\title{
Conhecimento sobre Hipertensão Arterial em pacientes acometidos pela doença no Município de Juazeiro do Norte - CE
}

\author{
Fábia Maria de Santana ${ }^{1}$, Danielly M. G. Matias ${ }^{2}$ Dallyane M. G. Matias ${ }^{3}$ Juliana de Freitas Vasconcelos ${ }^{4}$, \\ Hidembrugo Gonçalves Rocha
}

Resumo: A Hipertensão Arterial Sistêmica (HAS) é um problema grave de saúde pública no Brasil e no mundo. Ela é um dos mais importantes fatores de risco para o desenvolvimento de doenças cardiovasculares, cérebrovasculares e renais. O objetivo deste estudo foi avaliar o conhecimento sobre a hipertensão em pacientes acometidos pela doença, acompanhados por uma equipe da Estratégia Saúde da Família no município de Penaforte-Ce. Caracterizou-se por ser um estudo descritivo, com abordagem quantitativa, onde esta se fundamenta basicamente nas estatísticas para expressar os seus resultados, realizada em uma unidade básica do Município de Juazeiro do norte, os informantes da pesquisa foram portadores de Hipertensão Arterial Sistêmica, tendo um formulário semiestruturado como instrumento de coleta de dado, os dados foram analisados e apresentados em forma de tabelas e gráficos. Os resultados do nosso estudo evidenciaram que apesar das campanhas e ações educativas acerca dos riscos de uma pressão arterial descontrolada, uma pequena parcela destes clientes ainda demonstra pouco conhecimento sobre a patologia. Conclui-se, portanto, que muitos assuntos relacionados à hipertensão arterial ainda são desconhecidos por parte dos próprios portadores da doença.

Palavras chave: Idoso, Hipertensão arterial, Patologia

\section{Knowledge about Hypertension in patients affected by the disease in the city of Juazeiro do Norte - CE}

\begin{abstract}
Arterial Hypertension (HBP) is a serious public health problem in Brazil and worldwide. She is one of the most important risk factors for developing cardiovascular disease, cerebrovascular and renal. The aim of this study was evaluate the knowledge of hypertension in patients with the disease, accompanied by a team from the Family Health Strategy in the municipality of Penaforte-Ce. It was characterized by a descriptive and quantitative approach, where it is based primarily on statistics to express their results, performed in a basic unit of the northern city of Juazeiro, the informants in the study were suffering from Hypertension, having a form as semi-structured data collection instrument, data were analyzed and presented in tables and graphs. The results of our study showed despite campaigns and educational activities about the risks of uncontrolled blood pressure, a small portion of these customers still shows little knowledge of the pathology. We conclude, therefore, that many issues related to hypertension are still unknown from their own carriers.
\end{abstract}

Keywords: Elderly, Hypertension, pathology.

\footnotetext{
${ }^{1,2}$ Mestranda, Ciências da saúde, Faculdade de Medicina do ABC, Santo André, SP, Brasil

${ }^{3}$ Enfermeira, Pós graduada em gestão e assistência em saúde da família pela Faculdade de Juazeiro do norte-CE. E-mail: fabiagarban@hotmail.com

${ }^{4}$ Acadêmica de Medicina da Universidade Federal do Ceará. E-mail: julianafv@ hotmail.com

${ }^{5}$ Doutor em Farmacologia pela UFC. Professor da Faculdade de Medicina da Universidade Federal do Ceará - Barbalha, Ceará, Brasil. Email: hidemburgo.rocha@hotmail.com
} 


\section{Introdução}

A população mundial está envelhecendo, as mudanças socioeconômicas e os avanços técnicocientíficos do mundo contemporâneo têm oportunizado um incremento gradual na expectativa de vida da população mundial. As pessoas vivem mais devido à melhoria de acesso à água potável, alimentos, serviços de saúde, imunização, saneamento básico.

Tais ações têm viabilizado o aumento na expectativa de vida, porém, essas pessoas estão vivendo bem, satisfeitas e contentes com o que tem sob a ótica individual e coletiva? O que se percebe empiricamente é o contrário. As pessoas vivem mais, porém de forma precária e sofrida, sendo portadoras de doenças crônicas.

As pessoas idosas frequentemente apresentam uma ou mais doenças crônicas que são controladas com inúmeros medicamentos e complicadas por episódios agudos periódicos. Doenças crônicas não transmissíveis é um grupo de doenças com história natural prolongada, caracterizada por multiplicidade de fatores de risco complexos; interação de fatores etiológicos desconhecidos; longo período de latência; longo curso assintomático; manifestações clínicas, em geral de curso crônico, com períodos de remissão e exacerbação e evolução para incapacidades (REINERS et al, 2005).

A Hipertensão Arterial Sistêmica (HAS) é um problema grave de saúde pública no Brasil e no mundo. Ela é um dos mais importantes fatores de risco para o desenvolvimento de doenças cardiovasculares, cerebrovasculares e renais, sendo responsável por pelo menos $40 \%$ das mortes por acidente vascular cerebral, por $25 \%$ das mortes por doença arterial coronariana e, em combinação (BRASIL, 2006).

A falha no seguimento do tratamento sob o olhar exclusivo do profissional de saúde, caracterizando falta de adesão e consequente alteração da pressão arterial, consiste, empiricamente, em um problema aparentemente frequente e esta associada a vários fatores. Como o conhecimento popular daquele paciente pode interferir muito na sua adesão ao tratamento.

Diante de uma doença crônica na família, como e o caso da hipertensão arterial sistêmica (HAS), esta se comporta de acordo com sua representação que seja saúde-doença, evidenciando saberes diverso acerca da cura, que podem muitas vezes contrapor-se aos saberes médicos (SARAIVA et al, 2007).

De modo complementar, sabe-se que as crenças de acordo com as quais as pessoas tendem a viver afetam diretamente aos hipertensos na forma como enfrentam a doença e realizamos tratamento dessa enfermidade (PIRES e MUSSI,, 2008).

A educação continuada é um dos pilares do tratamento da hipertensão arterial, e para que exista um processo educativo eficaz e uma maior efetividade no atendimento, é necessário conhecer a 
atitude do individuo a respeito da doença da qual é portador. Muitas vezes, os costumes sobre as práticas de saúde, os valores e as percepções do paciente em relação à doença ou ao tratamento são diferentes daqueles pensados pelos profissionais da saúde, já que pertencem a grupos socioculturais, linguísticos e psicológicos distintos.

Nesse contexto, o interesse pala temática advém da vivência em estágios curriculares, no que foi possível perceber que durante o atendimento ainda existe uma grande carência, entre o cliente e profissional assim não induzido uma boa assistência.

Este estudo é relevante, uma vez que pode facilitar aos profissionais de saúde a obter informações e conhecimentos sobre as carências que os clientes hipertensos possuem referente a conhecimento de sua patologia. Dessa forma a equipe de saúde poderá intervir nessa realidade, podendo o cliente hipertenso vir a ser beneficiado com a melhoria na qualidade da assistência.

\section{Método}

Esse estudo foi do tipo descritivo, com abordagem quantitativa, onde esta se fundamenta basicamente nas estatísticas para expressar os seus resultados, o estudo descritivo vai oportunizar a descrição dentro do assunto ${ }^{5}$. Esse estudo foi conduzido nos meses de maio e junho de 2013, e realizado na Unidade Básica de Saúde (UBS) na qual atua a equipe 58 da Estratégia de Saúde da Família (ESF), localizada na Rua Arnóbio Bacelar Caneca, S/N no bairro Jardim Gonzaga da cidade de Juazeiro do Norte- CE.

A equipe 58 da ESF do município de Juazeiro do Norte - CE possuía até o presente momento da coleta de dados desta pesquisa 237 hipertensos cadastrados, sendo que somente 138 destes são acompanhados pela equipe os quais serão a amostra desta pesquisa, os demais são acompanhados por intermédios de convênios e/ou consultas particulares.

Para realizar a coleta de dados, foi utilizado um formulário semi-estruturado contendo questões inerentes ao objetivo proposto para a referida pesquisa e utilizado do modelo aplicado em um estudo realizado por Pierin (2001).

Para a realização desta pesquisa foi considerado o que preceitua a Resolução 196/96 CNS/MS, que versa sobre os aspectos éticos da pesquisa envolvendo seres humanos. ${ }^{7}$

\section{Análise e Discussão dos Resultados}

Id en lime Revista de Psicologia. Ano 8, No. 22, Fevereiro/2014 - ISSN 1981-1179. Edição eletrônica em http://idonline.emnuvens.com.br/id 
Este estudo buscou analisar o perfil e o conhecimento dos hipertensos usuários da Atenção Básica à Saúde sobre a Hipertensão Arterial Sistêmica. Os 97 clientes entrevistados responderam a perguntas que abordaram fatores como o ato de fumar, ingesta alcoólica, prática de atividades físicas e outras, que buscaram sondar o conhecimento dos entrevistados sobre o que é a patologia, os riscos que esta traz e os fatores que podem contribuir para o seu surgimento ou agravo.

Perfil dos clientes hipertensos usuários de uma Unidade Básica de Saúde. Juazeiro do Norte CE

Observa-se através da tabela 1 que dos 97 entrevistados, 37 pessoas $(38,1 \%)$ são do sexo masculino e $60(68,9 \%)$ são do sexo feminino. Estes, em sua maioria, encontrava-se com 60 anos ou mais de idade, totalizando 59 pessoas $(60,9 \%)$. A média da idade é de 61,7 anos com um desvio padrão $\mathrm{DP}=13,5$.

Tabela 1 - Perfil socioeconômico dos clientes hipertensos usuários de uma Unidade Básica de Saúde (ESF 58) de Juazeiro do Norte - CE. Mai/Jun 2013

\begin{tabular}{|c|c|c|c|c|c|c|}
\hline \multicolumn{7}{|c|}{ PERFIL SOCIOECONÔMICO DOS CLIENTES HIPERTENSOS } \\
\hline VARIÁVEIS & \multicolumn{4}{|c|}{ SEXO } & \multicolumn{2}{|c|}{ TOTAL } \\
\hline \multirow[b]{2}{*}{ FAIXA ETÁRIA } & \multicolumn{2}{|c|}{$\mathbf{M}$} & \multicolumn{2}{|c|}{$\mathbf{F}$} & \multirow{2}{*}{$\mathbf{n}$} & \multirow{2}{*}{$\%$} \\
\hline & $\mathbf{N}$ & $\%$ & $\mathbf{N}$ & $\%$ & & \\
\hline 26 a 29 anos & 01 & 2,7 & 02 & 3,3 & 03 & 3,1 \\
\hline 30 a 39 anos & 01 & 2,7 & 03 & 5,0 & 04 & 4,1 \\
\hline 40 a 49 anos & 03 & 8,1 & 09 & 15,0 & 12 & 12,4 \\
\hline 50 a 59 anos & 03 & 8,1 & 16 & 26,7 & 19 & 19,6 \\
\hline 60 a 69 anos & 13 & 35,1 & 18 & 30,0 & 31 & 32,0 \\
\hline 70 anos ou mais & 16 & 43,2 & 12 & 20,0 & 28 & 28,9 \\
\hline TOTAL & 37 & 100,0 & 60 & 100,0 & 97 & 100,0 \\
\hline
\end{tabular}

Fonte: Pesquisa direta, 2013.

Quanto ao estado civil os mais frequente foram o casado (47,7\%) e o viúvo (24,7\%). Vale ressaltar que 34 pessoas $(35,1 \%)$ afirmaram nunca ter estudado e $41(42,3 \%)$ possuíam o ensino fundamental incompleto, o que se faz concluir que a maioria delas possui baixo nível de escolaridade. 
Torna-se também relevante o fato de que 70 pessoas $(72,2 \%)$ declararam ter uma renda mensal inferior a um salário mínimo, apresentando um baixo nível socioeconômico, conforme mostrado na tabela 2 .

Tabela 2 - Perfil socioeconômico dos clientes hipertensos usuários de uma Unidade Básica de Saúde (ESF 58) de Juazeiro do Norte - CE. Mai-Jun /2013

\begin{tabular}{|c|c|c|c|c|c|c|}
\hline \multirow[b]{2}{*}{ ESTADO CIVIL } & \multicolumn{2}{|c|}{$\mathbf{M}$} & \multicolumn{2}{|c|}{$\mathbf{F}$} & \multirow[b]{2}{*}{$\mathbf{n}$} & \multirow[b]{2}{*}{$\%$} \\
\hline & $\mathbf{N}$ & $\%$ & $\mathbf{N}$ & $\%$ & & \\
\hline Casado & 18 & 48,6 & 28 & 46,7 & 46 & 47,4 \\
\hline Solteiro & 03 & 8,1 & 06 & 10,0 & 09 & 9,3 \\
\hline Separado / Divorciado & 05 & 13,5 & 12 & 20,0 & 17 & 17,5 \\
\hline Viúvo & 10 & 27,0 & 14 & 23,3 & 24 & 24,7 \\
\hline Outro & 01 & 2,7 & 00 & 0,0 & 01 & 1,0 \\
\hline \multirow[t]{2}{*}{ TOTAL } & 37 & 100,0 & 60 & 100,0 & 97 & 100,0 \\
\hline & \multicolumn{2}{|c|}{$\mathbf{M}$} & \multicolumn{2}{|c|}{$\mathbf{F}$} & & \\
\hline ESCOLARIDADE & $\mathbf{N}$ & $\%$ & $\mathbf{N}$ & $\%$ & $\mathbf{n}$ & $\%$ \\
\hline Não estudou & 16 & 43,2 & 18 & 30,0 & 34 & 35,1 \\
\hline Ensino Fundamental Incompleto & 14 & 37,8 & 27 & 45,0 & 41 & 42,3 \\
\hline Ensino Fundamental Completo & 04 & 10,8 & 04 & 6,7 & 08 & 8,2 \\
\hline Ensino Médio Incompleto & 00 & 0,0 & 03 & 5,0 & 03 & 3,1 \\
\hline Ensino Médio Completo & 03 & 8,1 & 05 & 8,3 & 08 & 8,2 \\
\hline Ensino Superior Incompleto & 00 & 0,0 & 03 & 5,0 & 03 & 3,1 \\
\hline \multirow[t]{2}{*}{ TOTAL } & 37 & 100,0 & 60 & 100,0 & 97 & 100,0 \\
\hline & \multicolumn{2}{|c|}{ M } & \multicolumn{2}{|c|}{$\mathbf{F}$} & & \\
\hline RENDA MENSAL & $\mathbf{N}$ & $\%$ & $\mathbf{N}$ & $\%$ & $\mathbf{n}$ & $\%$ \\
\hline Menos que um salário mínimo & 27 & 73,0 & 43 & 71,7 & 70 & 72,2 \\
\hline De um a três salários mínimos & 08 & 21,6 & 15 & 25,0 & 23 & 23,7 \\
\hline Acima de três salários mínimos & 02 & 5,4 & 02 & 3,3 & 04 & 4,1 \\
\hline TOTAL & 37 & 100,0 & 60 & 100,0 & 97 & 100,0 \\
\hline
\end{tabular}

Fonte: Pesquisa direta, 2013.

Quanto a cor da pele, a tabela 3 mostra que 42 dos hipertensos $(43,3 \%)$ são brancos, 48 são negros $(49,5 \%)$ e sete são pardos $(7,2 \%)$. Vale ressaltar que as pessoas que se declararam mestiças foram inclusas na categoria negra. Sobre a variável raça, existem várias teorias científicas que sustentam a tese que pessoas da raça negra têm maior suscetibilidade de adquirir Hipertensão Arterial Sistêmica. 
Tabela 3 - Distribuição quanto à cor da pele dos clientes hipertensos usuários de uma Unidade Básica de Saúde (ESF 58) de Juazeiro do Norte - CE. Mai-Jun /2013.

\begin{tabular}{l|c|c|c|c|c|c}
\hline \multirow{2}{*}{ COR DA PELE } & \multicolumn{2}{|c|}{ M } & \multicolumn{2}{|c|}{ F } & \multirow{2}{*}{} & \multirow{2}{*}{ ( } \\
\cline { 1 - 6 } Branca & $\mathbf{n}$ & $\mathbf{\%}$ & $\mathbf{N}$ & $\mathbf{\%}$ & $\mathbf{n}$ & $\mathbf{\%}$ \\
\hline Negra & 14 & 37,8 & 28 & 46,7 & 42 & 43,3 \\
\hline Parda & 22 & 59,5 & 26 & 43,3 & 48 & 49,5 \\
\hline TOTAL & 01 & 2,7 & 06 & 10,0 & 07 & 7,2 \\
\hline
\end{tabular}

Fonte: pesquisa direta, 2013.

Nos estudos de hipertensão arterial, o uso da variável raça como atributo essencial para definir populações, toma como base uma hipótese apoiada em dois pressupostos: a existência de um grupo de alelos que levariam a uma maior suscetibilidade à hipertensão, havendo uma maior prevalência destes alelos entre as pessoas negras quando comparadas às brancas, e a outra hipótese seria a associação entre a pigmentação da pele e a hipertensão, sugerindo que genes envolvidos na biossíntese da melanina também estivessem envolvidos nos mecanismos que produzem o aumento da pressão arterial, sugerindo com isto que quanto mais escura a pele, maior a presença desses alelos de suscetibilidade (LAGUARDIA, 2005).

Prosseguindo com a caracterização socioeconômica dos entrevistados, observou-se que estes são, em maior percentual, mulheres, casadas, acima de 60 anos com baixo nível econômico e educacional.

Este estudo vai ao encontro de Pereira et al (2007). quando estes também trazem uma maioria do sexo feminino e estado civil casado. Em seu estudo, estes afirmam ainda que 52,8\% dos seus entrevistados tinham ensino fundamental incompleto ou completo e que a maioria da população tinha uma baixa renda mensal. Os autores afirmam que a prevalência de Hipertensão Arterial Sistêmica (HAS) tende a ser maior nas classes mais baixas, onde os aspectos biopsicossociais irão influenciar diretamente neste fator.

Em pesquisa sobre a frequência de hipertensão arterial e fatores associados realizada através de inquérito telefônico em todos os estados brasileiros, Ferreira et al (2009). afirma que: "a frequência de HAS aumentou com a idade, diminuiu com a escolaridade, foi maior entre indivíduos de pele negra e nos viúvos e foi menor entre os solteiros".

Cotta et al (2008). trazem resultados semelhantes aos citados e acrescentam que por identificar uma maioria de hipertensos idosos, de baixa escolaridade e com grandes restrições econômicas, deve- 
se dar grande importância a estes aspectos, levando-os em consideração, principalmente, na elaboração de estratégias de intervenção e cuidado à saúde.

Os entrevistados foram abordados quanto ao hábito de fumar e a ingestão de bebidas alcoólicas. Dentre eles, 15 (15,5\%) afirmaram ser fumantes e $12 \quad(12,4 \%)$ afirmaram ingerir bebidas alcoólicas. Destes, sete pessoas $(7,2 \%)$ têm os dois hábitos. Atribuindo atenção aos fumantes e exfumantes, estes totalizam 54 pessoas $(55,7 \%)$, e quanto aos que tem o hábito de ingerir bebidas alcoólicas e os que ingeriam anteriormente foram 53 pessoas $(54,6 \%)$, o que indica que a maioria dos entrevistados faz ou já fez uso destas substâncias. O gráfico 1 demonstra o cruzamento de dados entre as duas variáveis (cigarro $\mathrm{x}$ bebidas alcoólicas).

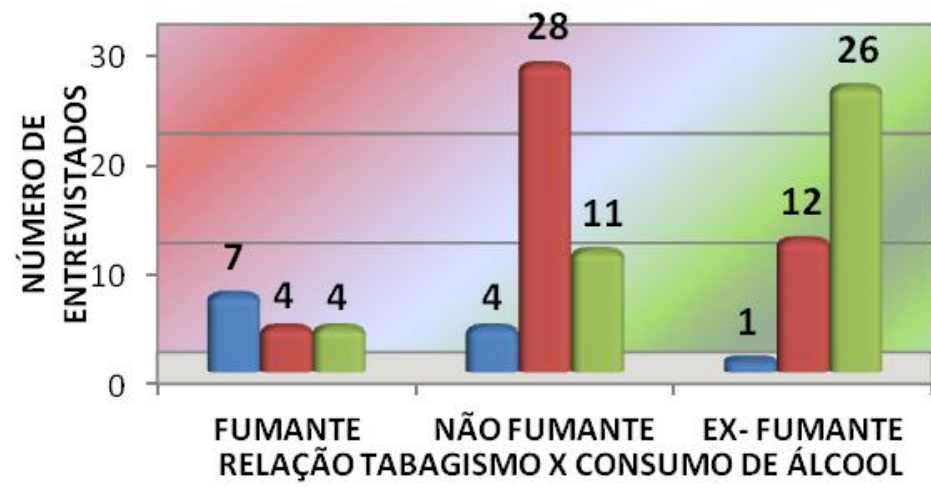

G INGEREÁLCOOL 口 NÃO INGERE ÁLCOOL G INGERIA ANTERIORMENTE

Gráfico 1 - Distribuição do número de clientes hipertensos usuários de uma Unidade Básica de Saúde (ESF 58) em relação às variável tabagismo e consumo de álcool. Juazeiro do Norte - CE. Mai-Jun /2013.

Fonte: Pesquisa direta, 2013.

O uso habitual de bebidas alcoólicas por pessoas hipertensas não é recomendado. O consumo moderado é tolerado por pacientes bem controlados. Recomenda-se limitar a ingestão de bebida alcoólica a menos de $30 \mathrm{ml} /$ dia de etanol para homens e a metade dessa quantidade para mulheres, preferencialmente com as refeições. Isso corresponde, para o homem, a ingestão diária de no máximo $720 \mathrm{ml}$ de cerveja (uma garrafa); $240 \mathrm{ml}$ de vinho (uma taça) ou $60 \mathrm{ml}$ de bebida destilada (uma dose). Aos pacientes que não conseguem se enquadrar nesses limites de consumo sugere-se o abandono do consumo de bebidas alcoólicas (BRASIL (2001)..

O hábito de fumar é outro fator a ser discutido. Este é um dos principais vilões das doenças cardiovasculares, além de ser prejudicial também a diversos outros sistemas.

Segundo o Ministério da saúde, o risco ocasionado pelo tabagismo é proporcional ao número de cigarros fumados e à profundidade da inalação, trazendo um risco ainda maior para as mulheres em 
relação aos homens. Os hipertensos fumantes devem ser estimulados a abandonar este ato (LAGUARDIA, 2005).

Neste estudo os ex-fumantes representam um grande grupo de 39 pessoas $(40,2 \%)$. Rosário et al (2009). afirmam que, geralmente, entre os hipertensos, o grupo de ex-fumantes é maior do que o de não fumantes. Este estudo não traz o mesmo resultado, porém traz números próximos entre os grupos. $\mathrm{O}$ número de ex-fumantes pode estar associado à conscientização e as campanhas educativas que relacionam o ato de fumar a HAS e aos riscos cardiovasculares.

Outro fator associado à prevalência de hipertensão nas mulheres é o uso de anticoncepcionais orais. Devido a este fato, interrogou-se entre as entrevistadas se estas fazem ou já fizeram uso de anticoncepcionais hormonais. Das 60 entrevistadas, 32 (53,4\%) fazem ou já fizeram uso destes contraceptivos. Os percentuais foram demonstrados através do gráfico 2.

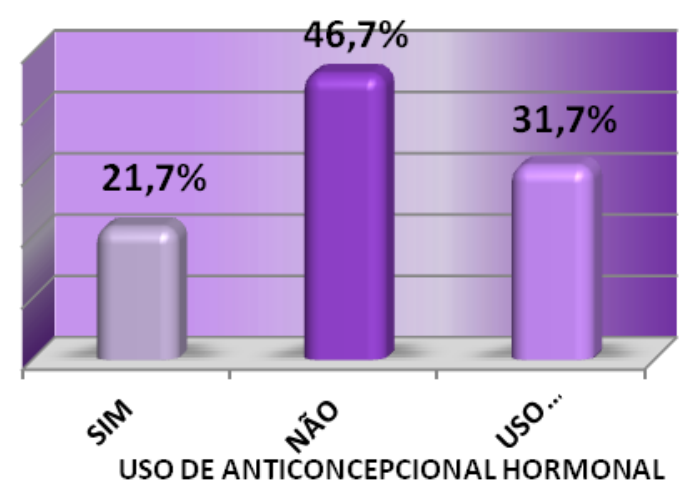

Gráfico 2 - Distribuição do percentual de mulheres hipertensas usuários de uma Unidade Básica de Saúde (ESF 58) em relação ao uso de contraceptivos hormonais. Juazeiro do Norte - CE. Mai-Jun /2013

Fonte: pesquisa direta, 2013.

Nas mulheres que usam anticoncepcionais orais, a hipertensão é de duas a três vezes mais comum do que naquelas que não usam. As mulheres que além do uso do contraceptivo oral, possuem idade avançada e são obesas trazem mais riscos de adquirir a doença. Em mulheres com mais de 35 anos e fumantes, o uso de anticoncepcional oral está contra-indicado (BRASIL, 2006).

Rosa, Plavnik e Tavares (2006), afirmam que embora o risco de hipertensão relacionado ao uso de contraceptivos orais seja baixo, o aparecimento de hipertensão por usuárias destes métodos deve ser investigado, suspendendo o medicamento por pelo menos três meses e monitorando os níveis pressóricos.

Existem fatores que ajudam a diminuir o risco de doenças cardiovasculares e também auxiliam no controle da pressão arterial. Dentre estes se destaca a prática de atividades físicas 
Os entrevistados foram abordados quanto ao ato de praticar alguma atividade física. Os resultados estão dispostos na tabela 4 . Deve-se observar que a maioria, 55 pessoas $(56,7 \%)$ declarou não realizar nenhuma atividade física. As pessoas sedentárias estão mais propensas ao aumento da pressão arterial.

Tabela 4 - Distribuição dos clientes hipertensos usuários de uma Unidade Básica de Saúde (ESF 58) quanto à prática de atividades físicas. Juazeiro do Norte - CE. Mai-Jun /2013

\begin{tabular}{|c|c|c|c|c|c|c|}
\hline \multicolumn{7}{|c|}{ PRÁTICA DE ATIVIDADES FÍSICAS } \\
\hline \multirow[b]{2}{*}{ VARIÁVEIS } & \multicolumn{2}{|c|}{$\mathbf{M}$} & \multicolumn{2}{|r|}{$\mathbf{F}$} & \multirow[b]{2}{*}{$\mathbf{n}$} & \multirow[b]{2}{*}{$\%$} \\
\hline & $\mathbf{n}$ & $\%$ & $\mathbf{n}$ & $\%$ & & \\
\hline SIM & 13 & 35,1 & 21 & 35,0 & 34 & 35,1 \\
\hline $\mathrm{NÃO}$ & 21 & 56,8 & 34 & 56,7 & 55 & 56,7 \\
\hline PRATICAVA & & & & & & \\
\hline ANTERIORMENTE & 03 & 8,1 & 05 & 8,3 & 08 & 8,2 \\
\hline TOTAL & 37 & 100,0 & 60 & 100,0 & 97 & 100,0 \\
\hline
\end{tabular}

Fonte: pesquisa direta, 2013.

Os profissionais de saúde que estão diretamente em contato com os clientes hipertensos devem buscar a adesão desses a alguma atividade física, explicando os benefícios que esta pode trazer não só para o controle da pressão arterial como para o bem-estar físico e mental do cliente. O gráfico 3 mostra as atividades citadas pelos 42 entrevistados $(43,3 \%)$ que afirmaram realizar ou já ter realizado alguma atividade física.

Na pesquisa de Cunha et AL (2008). é relatado que diante do grande número de pessoas sedentárias torna-se importante identificar os determinantes da atividade física, para que se possa propor programas de incentivo a esta prática, bem como estratégias que estimulem a população a adotar um estilo de vida fisicamente ativo.

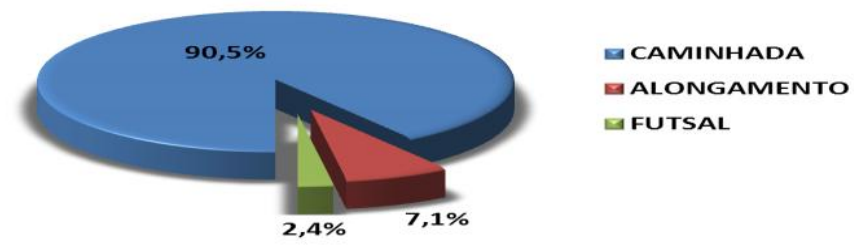

Gráfico 3 - Distribuição do percentual de atividades físicas relatadas pelos clientes hipertensos usuários de uma Unidade Básica de Saúde (ESF 58). Juazeiro do Norte - CE.Mai-Jun/2013. Fonte: pesquisa direta, 2013. 
Brasil (2001) traz recomendações que os profissionais devem considerar ao indicar a prática de atividades físicas: devem ser regulares, pois seus benefícios possuem ação máxima de apenas 24 a 48 horas; devem ser exercícios aeróbicos (caminhar, nadar, andar de bicicleta, etc.), pois os exercícios isométricos não são recomendados, a duração média deve ser de 45 a 60 minutos; no mínimo três vezes por semana [...]; a idade, a aptidão física e as preferências de cada paciente são importantes; os exercícios devem ser realizados com roupas e calçados adequados; deve-se ingerir líquidos em quantidade suficiente antes, durante e após o exercício, para evitar a desidratação [...].

Tabela 5 - Distribuição dos clientes hipertensos usuários de uma Unidade Básica de Saúde (ESF 58) quanto ao histórico familiar de hipertensão. Juazeiro do Norte - CE. Mai-Jun/2013.

\begin{tabular}{l|c|c}
\hline \multicolumn{3}{c}{ HISTÓRIA FAMILIAR DE HIPERTENSÃO } \\
\hline VARIÁVEIS & N & \% \\
\hline SIM & 57 & 58,8 \\
\hline NÃO & 40 & 41,2 \\
\hline TOTAL & $\mathbf{9 7}$ & $\mathbf{1 0 0 , 0}$ \\
\hline PARENTESCOS CITADOS: & $\mathbf{N}$ & $\mathbf{\%}$ \\
\hline PAI/MÃE & 46 & 80,7 \\
\hline IRMÃO(S) & 11 & 19,3 \\
\hline TOTAL & $\mathbf{5 7}$ & $\mathbf{1 0 0 , 0}$ \\
\hline
\end{tabular}

Fonte: pesquisa direta, 2013.

Ao se interrogar quanto à existência de hipertensos na família, 57 entrevistados $(58,8 \%)$ afirmaram que sim e citaram o parentesco conforme exposto na tabela 5. Deve-se ressaltar que a hereditariedade também é um dos fatores de risco para a HAS. Estes resultados concordam com Costa et al (2007). que em seu estudo sobre a prevalência de hipertensão arterial em adultos na cidade de Pelotas (RS), constataram associação entre a hipertensão e a história familiar do cliente, onde também houve uma prevalência de familiares hipertensos.

Conforme Laguardia (2005),

O estudo da contribuição genética na hipertensão arterial assume que a identificação dos determinantes genéticos pode fornecer as pistas que elucidarão os processos envolvidos na patogênese desse fenótipo e identificar os indivíduos com predisposição específica herdada. 
O fato de existir a predisposição genética à hipertensão deve ser utilizado como estratégias de prevenção e promoção de saúde, onde as ações educativas voltadas para a patologia possam esclarecer este fator e incentivar as pessoas saudáveis a realizarem cuidados com a saúde que possam contribuir para a prevenção da doença.

Quando não se consegue a manutenção dos níveis pressóricos através das modificações do estilo de vida, faz-se necessária a instituição da terapêutica farmacológica. Esta, durante o passar dos anos, tornou-se uma grande aliada no controle tanto da hipertensão quanto dos seus possíveis agravos (2005). Este estudo buscou identificar se os hipertensos fazem uso do tratamento medicamentoso, se estes utilizam algum tratamento alternativo e buscou, ainda, identificar a relação dos hipertensos com os dois tipos de tratamentos (tabela 6).

O principal objetivo do tratamento da hipertensão arterial é a redução da morbimortalidade cardiovascular do cliente hipertenso. Para tal propósito são utilizadas tanto medidas não farmacológicas isoladas como associadas a medicamentos anti-hipertensivos. Estes últimos, ao serem utilizados devem promover a redução dos níveis da pressão arterial e o risco de eventos cardiovasculares (PIERIN et al, 2001).

Tabela 6 - Distribuição dos clientes hipertensos usuários de uma Unidade Básica de Saúde (ESF 58) quanto à adesão do tratamento medicamentoso e outros tratamentos alternativos. Juazeiro do Norte CE.Mai-Jun/2013.

TRATAMENTO MEDICAMENTOSO X TRATAMENTOS ALTERNATIVOS

\begin{tabular}{|c|c|c|c|c|}
\hline \multirow[b]{2}{*}{ VARIÁVEIS } & & \multicolumn{2}{|c|}{ TRATAMENTOS ALTERNATIVOS } & \multirow{2}{*}{ TOTAL } \\
\hline & & SIM & NÃO & \\
\hline \multirow{2}{*}{$\begin{array}{l}\text { TRATAMENTO } \\
\text { MEDICAMENTOSO }\end{array}$} & SIM & $\begin{array}{c}58 \\
98,3 \%\end{array}$ & $\begin{array}{c}25 \\
65,8 \%\end{array}$ & $\begin{array}{c}83 \\
85,6 \%\end{array}$ \\
\hline & NÃO & $\begin{array}{c}01 \\
1,7 \% \\
\end{array}$ & $\begin{array}{c}13 \\
34,2 \% \\
\end{array}$ & $\begin{array}{c}14 \\
14,4 \% \\
\end{array}$ \\
\hline & TOTAL & $\begin{array}{c}59 \\
100,0 \%\end{array}$ & $\begin{array}{c}38 \\
100,0 \%\end{array}$ & $\begin{array}{c}97 \\
100,0 \%\end{array}$ \\
\hline
\end{tabular}

Fonte: pesquisa direta, 2013.

Verifica-se que 59 hipertensos $(60,8 \%)$ afirmaram realizar algum tratamento alternativo e 83 hipertensos $(85,9 \%)$ afirmaram aderir ao tratamento medicamentoso. Destes, 58 pessoas $(59,8 \%)$ disseram realizar tanto o tratamento medicamentoso como outros tratamentos alternativos. Quando 
interrogadas sobre os tipos de tratamentos alternativos que realizavam, estas citaram, dentre outros, a dieta alimentar balanceada (com restrição de sal e gorduras) e o uso de chás medicinais.

Quando detectada precocemente e no estágio leve ou moderada, a Hipertensão Arterial Sistêmica pode ser tratada com medidas não farmacológicas, onde o profissional de saúde irá recomendar mudanças no estilo de vida do hipertenso, sugerindo a diminuição ou exclusão de hábitos prejudiciais e incorporando hábitos saudáveis no dia-a-dia do indivíduo (JARDIN et al, 2007).

O tratamento medicamentoso visa reduzir os níveis pressóricos daqueles clientes que não obtiveram êxito somente com as mudanças no estilo de vida. Este pode ser instituído por uma única droga ou ainda pela associação de dois ou mais fármacos, que irão agir por mecanismos diferentes com um único objetivo: diminuir e estabilizar a pressão arterial (BRASIL, 2006).

Quanto aos hipertensos aderirem ou não ao tratamento anti-hipertensivo (PIRES e MUSSI, 2008).

\begin{abstract}
A adesão ao tratamento da hipertensão arterial vem sendo considerada um processo comportamental complexo, fortemente influenciado pelo meio ambiente, pelo indivíduo, pelos profissionais de saúde e pelos cuidados de assistência médica e perpassado por dimensões biológicas, socioeconômicas, psicológicas e culturais.
\end{abstract}

Segundo os mesmos autores, a Organização Mundial de Saúde afirma que a não adesão ao tratamento hipertensivo está diretamente ligada a fatores como: analfabetismo, baixo poder aquisitivo, desemprego, fornecimento de medicamentos limitados e o alto custo destes. Quanto ao presente estudo, observa-se uma adesão, pela maioria dos clientes, não só ao tratamento medicamentoso, como também a tratamentos alternativos, o que pode sugerir que apesar das dificuldades socioeconômicas, estes possuem uma assistência à saúde qualificada, que oferta o devido suporte necessário para a realização do tratamento (REINERS, et al 2005).

\title{
O conhecimento dos clientes hipertensos usuários de uma Unidade Básica de Saúde sobre a Hipertensão Arterial Sistêmica (HAS). Juazeiro do Norte - CE
}

Os entrevistados foram abordados sobre o conhecimento que detêm acerca da Hipertensão Arterial Sistêmica (HAS), sendo esta interrogada a eles através da expressão "pressão alta", buscando assim clareza e um melhor entendimento das perguntas realizadas. Seus resultados estão expostos na tabela 7. 
Tabela 7 - Distribuição das perguntas direcionadas aos clientes hipertensos usuários de uma Unidade Básica de Saúde (ESF 58) quanto ao conhecimento acerca da Hipertensão Arterial Sistêmica. Juazeiro do Norte - CE.Mai-Jun/2013

\begin{tabular}{|c|c|c|c|}
\hline \multicolumn{4}{|c|}{$\begin{array}{c}\text { CONHECIMENTO DOS ENTREVISTADOS SOBRE A } \\
\text { HIPERTENSÃO ARTERIAL SISTÊMICA }\end{array}$} \\
\hline \multicolumn{2}{|l|}{ PERGUNTA 1: } & n & $\%$ \\
\hline \multirow{3}{*}{$\begin{array}{l}\text { Você acha que pressão alta traz } \\
\text { complicações? }\end{array}$} & SIM & 94 & 96,9 \\
\hline & $\mathrm{NÃO}$ & 03 & 3,1 \\
\hline & TOTAL & 97 & 100,0 \\
\hline \multicolumn{2}{|l|}{ PERGUNTA 2: } & $\mathbf{n}$ & $\%$ \\
\hline \multirow{3}{*}{$\begin{array}{l}\text { Você acha que pressão alta é para } \\
\text { toda a vida? }\end{array}$} & SIM & 66 & 68,0 \\
\hline & $\mathrm{NÃO}$ & 31 & 32,0 \\
\hline & TOTAL & 97 & 100,0 \\
\hline \multicolumn{2}{|l|}{ PERGUNTA 3: } & $\mathbf{n}$ & $\%$ \\
\hline \multirow{3}{*}{$\begin{array}{l}\text { Você acha que pressão alta tem } \\
\text { cura? }\end{array}$} & SIM & 37 & 38,1 \\
\hline & $\mathrm{NÃO}$ & 60 & 61,9 \\
\hline & TOTAL & 97 & 100,0 \\
\hline \multicolumn{2}{|l|}{ PERGUNTA 4: } & $\mathbf{n}$ & $\%$ \\
\hline \multirow{3}{*}{$\begin{array}{l}\text { Você acha que pressão alta é uma } \\
\text { doença grave? }\end{array}$} & SIM & 82 & 84,5 \\
\hline & NÃO & 15 & 15,5 \\
\hline & TOTAL & 97 & 100,0 \\
\hline
\end{tabular}

Fonte: pesquisa direta, 2013.

Pôde-se notar que 94 clientes (96,9\%) acreditam que a hipertensão traz complicações. Foram 66 clientes $(68,0 \%)$ que afirmaram que a hipertensão é para toda a vida, ao passo em que 60 clientes $(61,9 \%)$ disseram acreditar que esta não tem cura. Sobre a gravidade da doença, 82 clientes $(84,5 \%)$ afirmaram que a pressão alta é uma doença grave. Observa-se que a maioria dos hipertensos tem o conhecimento básico da patologia, pois identificaram a cronicidade da doença, a sua gravidade (quando não tratada) e o fato de esta poder acarretar complicações cardiovasculares.

Apesar da resposta da maioria ser satisfatória, nota-se, ao cruzarem-se as respostas das questões entre si que há um desencontro entre as informações por parte alguns entrevistados.

\section{CRUZAMENTO DAS PERGUNTAS 01 E 04}

Id en line Revista de Psicologia. Ano 8, No. 22, Fevereiro/2014 - ISSN 1981-1179. Edição eletrônica em http://idonline.emnuvens.com.br/id 


\begin{tabular}{|c|c|c|c|c|}
\hline \multirow{4}{*}{\multicolumn{2}{|c|}{ PERGUNTAS: }} & \multirow{3}{*}{\multicolumn{2}{|c|}{$\begin{array}{c}\text { VOCÊ ACHA QUE } \\
\text { PRESSÃO ALTA É } \\
\text { UMA DOENÇA } \\
\text { GRAVE? }\end{array}$}} & \multirow{5}{*}{$\begin{array}{c}\text { TOTAL } \\
\\
\\
94\end{array}$} \\
\hline & & & & \\
\hline & & & & \\
\hline & & \multirow{3}{*}{$\begin{array}{c}\text { SIM } \\
81 \\
98,8 \%\end{array}$} & \multirow{3}{*}{$\begin{array}{c}\text { NÃO } \\
13 \\
86,7 \%\end{array}$} & \\
\hline \multirow{4}{*}{$\begin{array}{c}\text { VOCÊ ACHA QUE } \\
\text { PRESSÃO ALTA TRAZ } \\
\text { COMPLICAÇÕES? }\end{array}$} & \multirow{2}{*}{ SIM } & & & \\
\hline & & & & $96,9 \%$ \\
\hline & \multirow{2}{*}{ NÃO } & 01 & 02 & 03 \\
\hline & & $1,2 \%$ & $13,3 \%$ & $3,1 \%$ \\
\hline & TOTAL & $\begin{array}{c}82 \\
100,0 \%\end{array}$ & $\begin{array}{c}15 \\
100,0 \%\end{array}$ & $\begin{array}{c}97 \\
100,0 \%\end{array}$ \\
\hline
\end{tabular}

Quadro 1 - Cruzamento das perguntas 01 e 04 direcionadas aos clientes hipertensos usuários de uma Unidade Básica de Saúde (ESF 58) quanto ao conhecimento acerca da Hipertensão Arterial Sistêmica. Juazeiro do Norte - CE. Mai-Jun/2013. Fonte: pesquisa direta, 2013.

Ao cruzarem-se os dados das perguntas 01 e 04, observa-se que das 15 pessoas $(15,5 \%)$ que disseram não achar que a hipertensão é uma doença grave, duas $(2,1 \%)$ acham que além de não ser uma doença grave, esta também não traz complicações. Esta é uma minoria, porém significativa quando se trata da conscientização da dimensão dos problemas que a hipertensão pode ocasionar.

Apesar das campanhas e ações educativas acerca dos riscos de uma pressão arterial descontrolada, uma pequena parcela destes clientes ainda demonstra pouco conhecimento sobre a patologia. Torna-se mais preocupante ainda quando duas perguntas (02 e 03) que são realizadas consecutivamente, ambas abordando o mesmo assunto, porém de maneiras diferentes, trazem dúvidas e insegurança em suas respostas. Observe o quadro 2. 


\section{CRUZAMENTO DAS PERGUNTAS 02 E 03}

\begin{tabular}{|c|c|c|c|c|}
\hline \multirow[t]{2}{*}{ PERGUNTAS: } & & \multicolumn{2}{|c|}{$\begin{array}{c}\text { VOCÊ ACHA QUE } \\
\text { PRESSÃO ALTA TEM } \\
\text { CURA? }\end{array}$} & \multirow[t]{2}{*}{ TOTAL } \\
\hline & & SIM & NÃO & \\
\hline \multirow{2}{*}{$\begin{array}{c}\text { VOCÊ ACHA QUE } \\
\text { PRESSÃO ALTA É PARA } \\
\text { TODA A VIDA? }\end{array}$} & SIM & $\begin{array}{c}08 \\
21,6 \%\end{array}$ & $\begin{array}{c}58 \\
96,7 \%\end{array}$ & $\begin{array}{c}66 \\
68,0 \%\end{array}$ \\
\hline & NÃO & $\begin{array}{c}29 \\
78,4 \%\end{array}$ & $\begin{array}{c}02 \\
3,3 \%\end{array}$ & $\begin{array}{c}31 \\
32,0 \%\end{array}$ \\
\hline & TOTAL & $\begin{array}{c}37 \\
100,0 \%\end{array}$ & $\begin{array}{c}60 \\
100,0 \%\end{array}$ & $\begin{array}{c}97 \\
100,0 \%\end{array}$ \\
\hline
\end{tabular}

Quadro 2 - Cruzamento das perguntas 02 e 03 direcionadas aos clientes hipertensos usuários de uma Unidade Básica de Saúde (ESF 58) quanto ao conhecimento acerca da Hipertensão Arterial Sistêmica. Juazeiro do Norte - CE. Mai-Jun/2013. Fonte: pesquisa direta, 2013.

Nota-se que a maior parte dos entrevistados respondeu corretamente as perguntas, sendo esta os 58 clientes $(59,8 \%)$ que afirmaram que a hipertensão não tem cura e é uma doença para toda a vida, o que representa a sua cronicidade. Outros 29 clientes $(29,9 \%)$ afirmaram que a hipertensão tem cura e não é para toda a vida, demonstrando entender que esta não é uma doença crônica. Verifica-se ainda que oito clientes $(8,2 \%)$ responderam que a hipertensão é para toda a vida e que esta tem cura, simultaneamente, e outros dois $(2,1 \%)$ clientes afirmaram que a hipertensão não tem cura, mas também não é para toda a vida, o que pode ser interpretado como um não entendimento sobre a pergunta, ou ainda, sobre o assunto questionado.

As respostas que se voltam contra a cronicidade da doença e os $10,3 \%$ de clientes que demonstram um não entendimento tanto sobre a pergunta, como sobre a doença, também preocupa quanto ao nível de conhecimento deste grupo de pessoas.

Para se elaborar e implantar estratégias de cuidado à saúde de um grupo de pessoas, deve-se levar em consideração aspectos importantes, uma vez que há a necessidade de um trabalho mais específico para esse grupo populacional, que deve ser realizado de maneira clara e com o auxílio de recursos didáticos, atrativos e de fácil compreensão, visto que muitas dessas pessoas não obtiveram oportunidades de estudos e acesso a informações (JESUS et al, 2008). 


\begin{tabular}{|l|c|c|c|c|}
\hline \multicolumn{5}{|c|}{ FATORES RELACIONADOS À HIPERTENSÃO } \\
ARTERIAL SISTÊMICA \\
\cline { 2 - 5 } VARIÁ VEIS: & \multicolumn{3}{c|}{ SIM } & NÃO \\
\cline { 2 - 6 } & n & $\%$ & N & $\%$ \\
\hline SAL & 96 & 99,0 & 01 & 1,0 \\
\hline STRESS & 90 & 92,8 & 07 & 7,2 \\
\hline BEBIDA ALCOÓLICA & 92 & 94,8 & 05 & 5,2 \\
\hline FUMO & 88 & 90,7 & 09 & 9,3 \\
\hline ALIMENTOS GORDUROSOS & 90 & 92,8 & 07 & 7,2 \\
\hline EXCESSO DE PESO & 80 & 82,5 & 17 & 17,5 \\
\hline FALTA DE ATIVIDADE FÍSICA & 78 & 80,4 & 19 & 19,6 \\
\hline HEREDITARIEDADE & 63 & 64,9 & 34 & 35,1 \\
\hline AUMENTO DA IDADE & 39 & 40,2 & 58 & 59,8 \\
\hline USO DE ANTICONCEPCIONAIS & 29 & 29,9 & 68 & 70,1 \\
\hline SEXO DA PESSOA & 11 & 11,3 & 86 & 88,7 \\
\hline RAÇA & 14 & 14,4 & 83 & 85,6 \\
\hline
\end{tabular}

Quadro 3 - Distribuição dos fatores aos quais os clientes hipertensos usuários de uma Unidade Básica de Saúde (ESF 58) julgam estar relacionados ou não à Hipertensão Arterial Sistêmica. Juazeiro do Norte - CE. MaiJun/2013. Fonte: pesquisa direta, 2013.

São vários os estudos que expõem os diversos fatores de risco que influenciam no aparecimento ou agravo da hipertensão arterial (PEREIRA, 2007; COSTA, 2007; JESUS, 2008). Estes fatores estão expressos no quadro 3, onde foram apresentados aos clientes hipertensos, interrogando-os sobre o seu conhecimento acerca da relação dos fatores com o aumento da pressão arterial.

Observa-se no quadro que a maioria dos entrevistados afirmou que oito dos doze fatores tinham relação com a hipertensão. Fica expresso através dos resultados que fatores como o avançar da idade, o uso de anticoncepcionais, o sexo e a raça do indivíduo ainda são desconhecidos como agentes agravantes da hipertensão pela maioria. Alguns destes fatores de risco foram discutidos durante a dissertação desta análise.

Vale ressaltar que a prevalência da HAS aumenta com a idade. O maior risco de hipertensão nos indivíduos mais velhos se deve às alterações na musculatura lisa e no tecido conjuntivo dos vasos, como consequência do processo de envelhecimento (PEREIRA et al, 2007). 
As ações que envolvem a Atenção Básica à Saúde devem buscar um melhor controle dos clientes hipertensos, estabelecendo metas para ampliar o grau de conhecimento dos fatores de risco por esta população diretamente interessada, fornecendo mais informações para a prevenção do aparecimento destes fatores, buscando evitar complicações como as doenças cardiovasculares (LAGUARDIA et al, 2005).

Para que haja efetividade no tratamento da HAS, torna-se necessário que não só os clientes, mas a população em geral, conheçam estes fatores de risco e obtenham instruções e ferramentas que auxiliem na diminuição do número de casos da doença e na melhoria da qualidade de vida de todos.

\section{Considerações Finais}

A hipertensão arterial ainda é um assunto muito obscuro, embora muitos aspectos e questionamentos já tenham sido esclarecidos em relação a essa patologia. Mesmo assim esse assunto ainda apresenta-se amplo na visão do portador de hipertensão arterial.

Esse estudo marca sua relevância quando procura de maneira simples e concreta e objetiva entende e avaliar o conhecimento sobre a hipertensão entre pacientes acometidos pela doença; de acordo com esse objetivo conseguiu-se quantificar várias informações relevante tais como: foram 97 entrevistados dentre quais traçou-se o perfil onde foram identificados 37 homens e 60 mulheres. Quanto à cor da pele 42 são brancos, 48 negros e 07 pardos, ressaltando que pessoas que se declararam mestiças foram inclusas na categoria negra, o qual vem comprovar mais ainda a realidade descrita em várias literaturas, onde os negros tem maior suscetibilidade de desenvolver hipertensão.

Os entrevistados foram abordados quanto aos hábitos de vida de fumar e ingerir bebida alcoólica, resultando em 15,5\% pessoas afirmaram ser fumantes e 12,4\% afirmaram ingerir bebida alcoólica, os demais se incluem na categoria bebeu ou fumou anteriormente, ou ainda que nunca bebeu e nunca fumou. Mesmo assim ainda se torna preocupante o número de hipertensos que bebem ou fumam.

Um outro questionamento foi em relação ao uso de métodos contraceptivos hormonais entre as 60 mulheres entrevistadas, onde 21,7\% responderam sim, que fazem uso, 46,7\% que não fazem uso, e ainda $31,7 \%$ que já utilizaram anteriormente. No momento da coleta de dados ainda se torna perceptível a falta de conhecimento delas sobre a relação desses métodos com o risco de hipertensão arterial.

Sabe-se que existem vários fatores que ajudam a diminuir o risco de doenças cardiovasculares, dentre eles está a prática de atividade física. Nesse aspecto eles também foram questionados, e dentre 
os resultados: 34 indivíduos fazem prática de alguma atividade física, 55 não praticam atividade física, 08 praticaram anteriormente. Quanto ao uso de medicação, $98,3 \%$ responderam que fazem uso, e $1,7 \%$ relataram não fazerem uso de medicação.

Outro aspecto bastante relevante posto em questão foi sobre o conhecimento desses portadores de hipertensão arterial sobre sua patologia, onde a maioria deles demonstrou ter conhecimento acerca das complicações referentes ao aumento da pressão arterial; grande parte desses entrevistados também tem a consciência que a patologia é para toda vida, mas ao mesmo tempo se contradiz quando indagados se eles acham que a pressão alta é para toda vida, onde a maioria responde que não é para toda vida, e isso se contradiz com a pergunta anterior, permanece o questionamento ei eles não entenderam a pergunta feita ou se realmente eles não tem certeza na resposta dada. Quanto à gravidade da doença a maioria dos entrevistados responde que consideram a doença grave.

Levando em consideração os inúmeros fatores de risco relacionados à hipertensão arterial, percebe-se que a maioria dos entrevistados tem conhecimento da relação do sal, estresse, bebidas alcoólicas, fumo, alimentos gordurosos, excesso de peso, falta de atividade física, hereditariedade. Mas a maioria também ainda desconhece a relação da tendência que tem de aumento com a idade, com o uso de anticoncepcionais, e a relação com sexo da pessoa e a raça.

Conclui-se, portanto, que muitos assuntos relacionados à hipertensão arterial ainda são desconhecidos por parte dos próprios portadores da doença. Por isso os programas da Atenção Básica devem ser utilizados como meio de esclarecer esses conceitos de hipertensão arterial, uma vez que estes estão inteiramente relacionados como tratamento da patologia.

\section{Referências}

BRASIL, Ministério da Saúde, Conselho Nacional de Saúde. Comissão Nacional de Ética em Pesquisa. Resolução 196/1996 sobre pesquisas envolvendo seres humanos. Brasília: 1996.

BRASIL, Ministério da Saúde; Estatuto do Idoso. $2^{\text {a }}$ edição. Brasília: Editor Ministério da Saúde, 2006.

BRASIL. Ministério da Saúde. Departamento de Atenção Básica. Hipertensão Arterial Sistêmica (HAS) e Diabetes Mellitus (DM). Brasília: Ministério da Saúde, n. 11, 2001. 
COSTA, Juvenal Soares Dias da et al. Prevalência de Hipertensão Arterial em adultos e fatores associados: um estudo de base populacional urbana em Pelotas, Rio Grande do Sul, Brasil. Revista Arquivos Brasileiros de Cardiologia, v. 10, n.8, p. 95-110. 2007.

COTTA, Rosângela Minardi Mitre et al. Reflexões sobre o conhecimento dos usuários no contexto do Programa de Saúde da Família: a lacuna entre o saber técnico e o popular. Revista de Saúde Coletiva, v.18, n.4, p.745-766, 2008.

CUNHA, Lana Cândido et al. Fatores associados à prática de atividade física na população adulta de Goiânia: monitoramento por meio de entrevistas telefônicas. Revista Brasileira de Epidemiologia, v.11, n. 3, p. 495-504, 2008.

FERREIRA, Sandra R. Gouvea. Frequência de hipertensão arterial e fatores associados. Revista de Saúde Pública, v.43, n. 2, p.98-106, 2009.

JARDIM, Paulo César B. VEIGA et al. Hipertensão Arterial e alguns fatores de risco em uma capital brasileira. Ver Brasileira de Cardiologia. V. 6, n 1, pag. 51-62, 2007.

JESUS, Elaine dos Santos et al. Perfil de um grupo de hipertensos: aspectos biossociais, conhecimentos e adesão ao tratamento. Revista Acta Paulista de Enfermagem, v.21, n. 1, p. 59-65, 2008.

LAGUARDIA, Josué. Raça. Genética e hipertensão: nova genética ou velha eugenia? Revista História, Ciências, Saúde. Manguinhos. 2005. Disponível em: <http://www.scielo.br/pdf/hcsm/v12n2/07.pdf>. V. 2, n. 1, Pag. 21-28.

LIMA, Leonardo Miguel. Noções elementos da pesquisa cientificam. $4^{\mathrm{a}}$ ed. Rev. Atual Serra talhada, 2008.

LUZ, Madel T. Cultura contemporânea e medicinas alternativas: novos paradigmas em saúde no fim do século xx. Revista de Saúde Coletiva, v.15, n.1, p.145-176, 2005 
PEREIRA, Marcia Regina et al. Prevalência, conhecimento, tratamento e controle de hipertensão arterial sistêmica na população adulta urbana de Tubarão, Santa Catarina. Caderno de Saúde Pública, v. 23, n.10, p. 2363-2374, 2007.

PIERIN, A. M. G. et al. O perfil de um grupo de pessoas hipertensas de acordo com conhecimento e gravidade da doença. Rev Esc Enf, v.35, n. 1, p. 11-8, 2001.

PIRES, Cláudia Geovana da Silva; MUSSI, Fernanda Carneiro. Crenças em saúde para o controle da hipertensão arterial. Revista Ciência e Saúde Coletiva, v.13, n 2, p. 2257-2267, 2008.

REINERS, A. A. O.AZEVEDO R. C. S. et al. Adesão/não-adesão de pessoas ao tratamento de saúde. Revista Ciência \& Saúde Coletiva, v. 3, n. 2, P 156-198, 2005.

ROSA, E. Cantoni; PLAVNIK, F. Liane; TAVARES, Agostinho. Hipertensão Arterial Sistêmica. Revista Brasileira de Medicina, v. 8, n 1, p. 10-25, 2006.

ROSÁRIO, Tânia Maria do et al. Fatores associados à hipertensão arterial sistêmica em Nobres-MT. Revista Brasileira de Epidemiologia, v.12, n. 2, p. 248-257, 2009.

SARAIVA, K. R .O et al. Saber do familiar na adesão da pessoa hipertenso ao tratamento: analise com base na educação popular em saúde. Rev Texto contexto - enferm, v. 1, n. 2, Pag. 51-58, 2007.

\section{Como citar este artigo (Formato ISO):}

SANTANA, F.M.; MATIAS, D.M.G.; MATIAS, D.M.G.; VASCONCELOS, J.F.; ROCHA, H.G. Conhecimento sobre hipertensão arterial em pacientes acometidos pela doença no município de Juazeiro do Norte - CE.. Id on Line Revista de Psicologia, Fevereiro de 2014, vol.8, n.22, p. 182-201. ISSN 1981-1189. 